\title{
Equilibrium and stability of relativistic cylindrical polytropes
}

\author{
M. A. Scheel \\ Department of Physics and Center for Radiophysics and Space Research, Cornell University, Ithaca, New York 14853 \\ S. L. Shapiro and S. A. Teukolsky \\ Department of Physics, Center for Radiophysics and Space Research, \\ and Department of Astronomy, Cornell University, Ithaca, New York 14853
}

(Received 30 November 1992)

\begin{abstract}
We examine the structure and radial stability of infinitely long cylindrical polytropes in general relativity. We show that in contrast with spherical polytropes, all cylindrical polytropes are stable. Thus pressure regeneration is not decisive in determining the behavior of cylindrical systems. We discuss how the behavior of infinite cylinders is qualitatively different from that of finite, asymptotically flat configurations. We argue that the use of infinite cylinders to gain physical insight into the collapse of finite aspherical systems may be misleading. In particular, the ability of pressure and rotation to always halt the collapse of an infinite cylinder to a naked singularity may not carry over to finite systems.
\end{abstract}

PACS number(s): 04.20.Jb, 95.30.Sf

\section{INTRODUCTION}

Recent numerical calculations [1] have shown that a nonrotating prolate spheroid made up of collisionless particles can undergo gravitational collapse to a spindle singularity without the formation of an apparent horizon. The singularity extends along the axis from the matter interior into the vacuum exterior. An apparent horizon appears only if the collapsed spheroid is sufficiently compact in all of its spatial dimensions, so that $\mathcal{C} \lesssim 4 \pi$. Here $\mathcal{C}$ is the minimum circumference that completely surrounds the mass $M$ in any orientation (the hoop conjecture $[2,3])$. If the spheroid is sufficiently long, no apparent horizon forms. However, there is no guarantee that the absence of an apparent horizon in the numerical calculations implies the absence of an event horizon, although the converse is true. If, in fact, the spindle singularity forms without a black hole present, then the numerical example would provide a clear violation of Penrose's cosmic censorship conjecture [4]. According to cosmic censorship, any physical singularity formed during collapse must be "clothed" by an event horizon so that there can be no "naked" singularities. Only if this is the case can general relativity predict the future evolution, since then the region outside the horizon is causally disconnected from the singularity inside and cannot be influenced by it.

It is natural to ask whether the above example is special, or whether it illustrates some generic feature of highly nonspherical collapse in general relativity. We note that the simulation was performed with a nonrotating configuration of collisionless particles. If one includes rotation or treats fluid matter with hydrodynamic pressure, does a prolate spheroid still collapse to a singular state? Alternatively, does rotation or pressure halt the collapse before a singularity can form?

The answer to this question is not at all obvious. In Newtonian physics, angular momentum conservation prevents a rotating object from collapsing to a singularity along the rotation axis. In general relativity, however, when matter collapses to a Kerr black hole, a small amount of rotation clearly does not prevent the formation of a singularity in the strong field region inside the black hole. Likewise, in Newtonian physics, pressure can halt the collapse of a fluid, whereas in general relativity, high pressure can actually accelerate collapse to a black hole. This phenomenon arises from "pressure regeneration" [3] and shows that pressure does not necessarily present the formation of a singularity.

Attempts at understanding the effects of rotation and pressure in prolate collapse have been made by studying the extreme case of collapse of an infinitely long cylinder. An infinite cylinder of dust collapses to form a naked line singularity $[2,3,5]$ along the axis. This singularity is similar in some respects to the one formed by the collapse of the prolate spheroid [1]: In both cases the singularity is extended along one spatial dimension, rather than pointlike. Furthermore, in both cases the proper length of a fixed segment of matter grows slowly while its circumference and area shrink rapidly as the collapse progresses. Because of these geometric similarities, it has been suggested [6] that the behavior of cylindrical systems may illustrate the essential physics of prolate collapse and the formation of spindle singularities. In particular, the local evolution of a very long spindle away from its end points might be well approximated by that of an infinite cylinder.

However, there are some important differences between infinite cylinders and finite configurations. The metric associated with any finite system is asymptotically flat, whereas the metric of an infinite cylinder diverges as one moves far from the axis of symmetry. Black holes do not form from the collapse of infinite cylinders. Finite configurations (e.g., spheres) can undergo catastrophic gravitational collapse to black holes and singularities even in the presence of pressure and rotation. However, 
there is evidence that rotation or pressure may be sufficient to prevent the collapse of an infinite cylinder to a singularity. Apostolatos and Thorne [6] have shown that an infinite cylindrical shell made of an equal number of co- and counter-rotating particles cannot collapse to a singularity, even for small rotation. Piran [7] has constructed numerical examples of imploding infinite fluid cylinders that bounce at finite radii without collapsing to singularities. It may thus be the case that the collapse of an infinite cylinder is not always a reliable indicator of the collapse of a finite prolate system.

\section{Structure and stability of equilibrium polytropic cylinders}

In this paper, we consider whether pressure regeneration is ever important for infinite cylinders. We do this by examining the structure and radial stability of infinite cylinders supported in hydrostatic equilibrium by a polytropic equation of state:

$$
p=K \rho_{0}^{\Gamma} \text {. }
$$

Here $p$ is the pressure, $\rho_{0}$ is the rest mass density, and $K$ and $\Gamma$ are constants. The physically relevant range of values for $\Gamma$ is $1 \leq \Gamma \leq 2$. For $\Gamma<1$ the fluid is unstable against local microscopic collapse, even in the absence of gravitational forces. For $\Gamma>2$, the speed of sound can exceed the speed of light, violating causality.

In the spherical case, a Newtonian polytrope in hydrostatic equilibrium is stable with respect to radial pulsations if the adiabatic index $\Gamma$ is greater than $\Gamma_{c}=\frac{4}{3}$ and it is unstable if $\Gamma<\frac{4}{3}$. However, because of pressure regeneration, the critical adiabatic index $\Gamma_{c}$ for relativistic spherical polytropes is greater than $\frac{4}{3}$. In fact, any spherical equilibrium polytrope, regardless of the value of $\Gamma$, will be unstable with respect to radial perturbations if it is sufficiently relativistic [8]. In the case of an infinite cylinder, the critical index $\Gamma_{c}$ is equal to unity for a Newtonian polytrope. Because $\Gamma<1$ is unphysical, one can say that all equilibrium Newtonian cylindrical polytropes are stable with respect to radial perturbations. The corresponding situation for relativistic cylindrical polytropes has apparently not been analyzed. If pressure regeneration is important in cylinders, one would expect to find relativistic equilibrium polytropes that are unstable under the following conditions. First of all, moderately relativistic cylinders should become unstable as $\Gamma$ approaches unity. Second, all equilibrium cylindrical polytropes, regardless of the value of $\Gamma$, should become unstable as they become sufficiently relativistic.

In fact, we show in this paper that all relativistic polytropic cylinders are stable to radial perturbations. First we construct equilibrium configurations with various values of $\Gamma$, and we show using $C$-energy arguments that there is no quasinormal mode of oscillation that changes stability as the central energy density ranges from nonrelativistic to ultrarelativistic values. Since Newtonian cylinders are stable, this result implies that all cylinders are stable. However, this analysis does not consider the possibility that Newtonian cylinders can become unstable when one allows for the emission of gravitational radia- tion. (This possibility does not exist for spherical polytropes because of Birkhoff's theorem.) To verify that gravitational radiation cannot drive the instability, we explicitly solve Einstein's equations for small perturbations about equilibrium. Expressing the perturbation as a superposition of quasinormal modes, we find numerically that all the quasinormal modes are damped in time. This result shows that pressure regeneration does not destabilize infinite cylindrical systems, and explains the numerical results found by Piran [7]. In addition, our finding provides yet another example in which the behavior of infinite cylinders is qualitatively different from that of finite configurations.

\section{CYLINDRICAL GEOMETRY}

Much of the theory of cylindrical systems, including the concept of $C$ energy, has been developed by Thorne [9]. The following is a summary of the main results that are relevant to this paper.

A cylindrical system is one that is invariant under rotations about a symmetry axis and translations parallel to that axis. In any such system, one can choose coordinates $(t, r, z, \phi)$ such that $\phi$ is periodic with period $2 \pi$, and the metric tensor is independent of $z$ and $\phi$. They are two spacelike Killing vectors associated with a cylindrical spacetime, $\xi_{\phi}=\partial / \partial \phi$ and $\xi_{z}=\partial / \partial z$. The Killing vector $\xi_{\phi}$ is uniquely determined by the periodicity of $\phi$, but $\xi_{z}$ is only determined up to a transformation of the form

$$
\xi_{z^{\prime}}=a \xi_{z}+b \xi_{\phi} \quad(a \neq 0) .
$$

If a cylindrical system is also invariant under reflections in planes containing or perpendicular to the axis of symmetry, it is called whole-cylinder symmetric. In this case, it is possible to choose a coordinate system such that $\xi_{z}$ and $\xi_{\phi}$ are hypersurface orthogonal, and such that $\xi_{z} \xi_{\phi}=0$. In this case, the only arbitrariness remaining in $\xi_{z}$ (or equivalently in the coordinate $z$ ) is the overall scale. For any particular system, one must make a standard choice of this scale. Although this choice is arbitrary and does not affect the physics of a particular system, it is important to choose the standard length consistently when comparing different systems.

It is useful to define the circumferential radius $R$ at any point in cylindrical spacetime as the proper circumference of the invariant cylindrical surface passing through that point, divided by $2 \pi$. Equivalently, $R$ is the length of the Killing vector $\xi_{\phi}$. If a cylinder is in hydrostatic equilibrium, has positive central pressure, and satisfies $p \leq \rho$ everywhere (where $p$ and $\rho$ are the pressure and energy density in geometrized units), then $R$ is zero at the center of the cylinder and increases monotonically to infinity at infinite proper radius.

The general static whole-cylinder symmetric vacuum solution can be written in the Levi-Civita form $[9,10]$

$$
\begin{aligned}
d s^{2}= & \left(r / r_{0}\right)^{4 k(1+2 k)}\left(d t^{2}-d r^{2}\right)-\left(r / r_{0}\right)^{-4 k} d z^{2} \\
& -r_{0}^{2}\left(r / r_{0}\right)^{2+4 k} d \phi^{2} .
\end{aligned}
$$

The constant $k$ is known as the Levi-Civita mass parameter; in the Newtonian limit it reduces to the mass per unit 
length of the axis singularity. The conicality parameter $r_{0}$ determines the circumferences of circles in $z=$ const planes: changing $r_{0}$ while holding $k$ constant changes $g_{\phi \phi}$ by a scale factor. Just as the metric outside of a finite spherical star is given by the Schwarzschild solution, the metric outside of a cylindrical star is given by the LeviCivita solution, but in this case only if the interior solution is static, because a nonstatic cylindrical system can produce gravitational waves.

\section{$C$ energy}

$C$ energy, or cylindrical energy, a quantity first defined and interpreted by Thorne [9], is the cylindrical analogue of the Schwarzschild mass $M$ in spherical systems, and has many similar properties. For example, $C$ energy is minimized in stable equilibrium configurations and maximized in unstable equilibrium configurations, and $C$ energy reduces to rest mass in the Newtonian limit.

An important difference between $C$ energy and Schwarzschild mass is that although the vacuum region outside of a spherical star contributes nothing to the Schwarzschild mass, the vacuum outside of a cylindrical star does contain $C$ energy. It is for this reason that one must be careful in comparing the total $C$ energies (per unit standard length) of two cylindrical stars: One cannot simply consider the $C$ energy per unit standard length contained within each star because of the vacuum contribution, but neither can one include the entire vacuum contribution out to radial infinity because it diverges. Instead, one must choose an arbitrary but fixed circumferential radius $R_{b}$, and define the effective $C$ energy per unit standard length of a system as that contained within the surface defined by $R_{b}$.

The $C$-energy scalar $U$ can be defined in the following way: Every point $(t, r, z, \phi)$ in cylindrical spacetime lies in an invariant two-dimensional cylindrical surface defined by

$$
\{(t, r, z+\alpha, \phi+\beta): \alpha \in(0,1), \beta \in(0,2 \pi)\}
$$

If the area of this surface is $A$, and its gradient is $A_{, \mu}$, then

$$
U \equiv \frac{1}{8}\left[1+\frac{A_{, \mu} A^{, \mu}}{4 \pi^{2}\left|\xi_{z}\right|^{2}}\right],
$$

where $\xi_{z}$ is the standard $z$ Killing vector. The total $C$ energy per unit standard length within a cylindrical region is equal to the value of $U$ at the surface of the region.

Notice that the meaning of $U$ ( $C$ energy per unit standard length) depends on the arbitrarily chosen standard length scale of the $z$ coordinate, and therefore one must choose the standard length scale consistently when comparing the $C$ energies of different systems. The most useful way to do this is to fix the standard $z$ coordinate such that standard length is equal to proper length at $R=R_{b}$, the circumferential radius at the surface that defines the effective boundary of the system.

\section{DIAGNOSIS OF STABILITY USING $C$ ENERGY}

The concept of $C$ energy is quite useful in understanding hydrostatic equilibrium and stability of cylindrical systems. Some of the properties of $C$ energy relating to hydrostatic equilibrium and stability include the following [9].

(1) Consider a family of momentarily static, wholecylinder symmetric configurations of cold perfect fluid such that there is a fixed number of particles per standard coordinate length and that there exists an outer surface that lies within the two-surface $R=R_{b}$. Every such configuration that extremizes the $C$ energy per unit standard length inside $R=R_{b}$ is an equilibrium configuration, and has $\xi_{t} \xi_{z}=\xi_{t} \xi_{\phi}=0$. The converse is also true.

(2) Among the equilibrium configurations considered in the previous statement, those which minimize the $C$ energy per unit standard length inside $R=R_{b}$ are stable with respect to small radial perturbations, and those which maximize the $C$ energy are unstable.

(3) Consider two equilibrium configurations which differ slightly in central density. The difference in $C$ energy per unit standard length between the two configurations is related to the difference in the number of particles per unit standard length by

$$
\delta U_{b}=\left(\frac{R_{b}}{R_{s}}\right)^{-2 k} \mu_{s} \delta A
$$

where $\mu_{s}$ is the rest mass of a particle, $R_{s}$ is the circumferential radius at the surface of the cylinder, and $k$ is the Levi-Civita mass parameter of the external gravitational field.

(4) If one constructs a sequence of equilibrium configurations parametrized by the central energy density $\rho_{c}$, and one chooses $R_{b}$ to lie outside the surface of each configuration, then one of these configurations has a zero-frequency radial acoustic mode if and only if it lies at a critical point of $U_{b}\left(\rho_{c}\right)$ and at a critical point of $k\left(\rho_{c}\right)$. Furthermore, if one writes the central density of this critical configuration as $\rho_{c r}$, then configurations with $\rho_{c}<\rho_{c r}$ are stable if and only if $U_{b}^{\prime \prime}\left(\rho_{c r}\right) R_{s}^{\prime}\left(\rho_{c r}\right)>0$, and configurations with $\rho_{c}>\rho_{c r}$ are stable if and only if $U_{b}^{\prime \prime}\left(\rho_{c r}\right) R_{s}^{\prime}\left(\rho_{c r}\right)<0$, where primes denote differentiations with respect to $\rho_{c r}$.

It is the fourth property that is most useful for diagnosing stability of equilibrium configurations. Recall that in the spherical case, one can determine changes in stability by looking for turning points in a plot of Schwarzschild mass versus central energy density [8]; the fourth property above tells us that we can do almost the same thing in the cylindrical case: If we choose a value of $R_{b}$ and compute $U_{b}$ and $k$ (by integrating the equilibrium field equations) for a set of cylinders with different central energy densities $\rho_{c}$, then any transitions between stable and unstable equilibrium configurations are indicated by critical points in the graphs of $U_{b}\left(\rho_{c}\right)$ and $k\left(\rho_{c}\right)$. 


\section{Equilibrium equations for polytropes}

The general static line element for a whole-cylinder symmetric system can be written in the form

$d s^{2}=-e^{2 v} d t^{2}+e^{2(\gamma-\psi)} d r^{2}+e^{2 \psi} d z^{2}+r^{2} e^{-2 \psi} d \phi^{2}$,

where $v(r), \gamma(r)$, and $\psi(r)$ are chosen to vanish at $r=0$. With this choice of coordinates, the circumferential radius $R$ is given by

$$
R=r e^{-\psi} \text {. }
$$

Note that the $z$ coordinate in Eq. (7) is not the standard $z$ coordinate: $z$ coordinate length is equal to proper length at $R=r=0$ and not at $R=R_{b}$. Instead, one unit of standard length is equal to $e^{\psi_{b}}$ multiplied by one unit of $z$ coordinate length, where $\psi_{b}$ is the value of $\psi$ at $R_{b}$. The reason that the standard $z$ coordinate is not used in the line element is that $R_{b}$, which determines the standard normalization of $z$, must be chosen to be greater than the surface circumferential radius $R_{s}$ of each cylinder in the sequence of equilibrium models, and this radius $R_{s}$ is not known until one integrates the field equations.

It is useful to define the variable

$$
M \equiv \frac{1}{8}\left(1-e^{2(v+\psi-\gamma)}\right),
$$

in which case the static field equations (in geometrized units) for a perfect gas are given by [9]

$$
\begin{aligned}
\frac{d M}{d r} & =2 \pi r(\rho-p) e^{2 v}, \\
\frac{d \psi}{d r} & =\frac{1}{2 r}\left[1-(1-8 M)^{-1 / 2}\right], \\
\frac{d v}{d r} & =\frac{2}{r(1-8 M)}\left(M+4 \pi r^{2} e^{2 v} p\right), \\
\frac{d p}{d r} & =-\frac{d v}{d r}(p+\rho),
\end{aligned}
$$

subject to the boundary conditions

$$
\begin{aligned}
& M=\frac{d M}{d r}=\psi=\frac{d \psi}{d r}=v=\frac{d v}{d r}=\frac{d p}{d r}=0, \\
& p=p_{c} \text { at } r=0 .
\end{aligned}
$$

Here $\rho$ is the total energy density, and $p$ is the pressure. In terms of $\rho$, a polytropic equation of state of the form (1) can be written as

$$
\rho=(p / K)^{1 / \Gamma}+n p,
$$

where the polytropic index $n$ is equal to $1 /(\Gamma-1)$.

If the cylinder has a finite radius, the line element (7) can be smoothly joined to the exterior Levi-Civita solution. The resulting Levi-Civita parameters are given by [9]

$$
\begin{aligned}
& k=\frac{1}{4}\left[\left(1-8 M_{s}\right)^{-1 / 2}-1\right], \\
& r_{0}=r_{s} e^{-\psi_{s}}\left[\left(1-8 M_{s}\right)^{1 / 2} e^{-\left(v_{s}+\psi_{s}\right)}\right]^{(1+2 k) / 4 k^{2},},
\end{aligned}
$$

where the $s$ subscript denotes the value on the surface of the cylinder. In addition, the $C$ energy per unit standard length inside $r$ is given by [9]

$$
U=\frac{1}{8}\left[1-(1-8 M) e^{-2(v+\psi)}\right] .
$$

Since $U$ only takes on values between 0 and $\frac{1}{8}$, it may be difficult to locate critical points of $U_{b}\left(\rho_{c}\right)$ numerically, especially when $U_{b}$ is large. Notice, however, that the metric parameter $\gamma$, which takes on values between zero and infinity, is a monotonic increasing function of $U$ :

$$
\gamma \equiv-\frac{1}{2} \ln (1-8 U) \text {. }
$$

Thus critical points of $\gamma_{b}\left(\rho_{c}\right)$ coincide with critical points of $U_{b}\left(\rho_{c}\right)$, so one can use $\gamma_{b}$ in place of $U_{b}$ when diagnosing stability.

While we are interested in values of the metric functions at $R=R_{b}$, it is not necessary to integrate numerically all the way out to $R_{b}$ because the field equations (10) simplify considerably for $r \geq r_{s}$ :

$$
\begin{aligned}
& M=M_{s}=\text { const }, \\
& p=\rho=0, \\
& \nu(r)=\nu_{s}+\frac{2 M_{s}}{1-8 M_{s}} \ln \left(\frac{r}{r_{s}}\right), \\
& \psi(r)=\psi_{s}-2 k \ln \left(\frac{r}{r_{s}}\right) .
\end{aligned}
$$

Using these relations, it is possible to calculate $\gamma_{b}$ knowing only the surface parameters and $R_{b}$ :

$$
\gamma_{b}=-\frac{1}{2} \ln \left(1-8 M_{s}\right)+v_{s}+\psi_{s}+\frac{4 k^{2}}{1+2 k} \ln \left(\frac{R_{b}}{R_{s}}\right) \text {. }
$$

Therefore, the procedure for diagnosing stability is the following: Using different values of central density $\rho_{c}$, integrate the field equations (10) to the surface of each cylinder, defined as the point at which $p$ and $\rho$ vanish, and thus obtain the functions $R_{s}\left(\rho_{c}\right), \psi_{s}\left(\rho_{c}\right), M_{s}\left(\rho_{c}\right)$, $v_{s}\left(\rho_{c}\right)$, and $k\left(\rho_{c}\right)$. Then choose $R_{b}$ to be greater than the maximum value of $R_{s}\left(\rho_{c}\right)$ (we choose $R_{b}$ equal to twice this maximum value), and use Eq. (18) to obtain $\gamma_{b}\left(\rho_{c}\right)$. Any transition from stability to instability, or vice versa, is indicated by a critical point in the graph of $\gamma_{b}\left(\rho_{c}\right)$ that coincides with a critical point in the graph of $k\left(\rho_{c}\right)$.

\section{DETERMINATION OF COMPLEX EIGENFREQUENCIES}

Another method of determining whether an equilibrium cylindrical model is stable or unstable with respect to radial perturbations is to directly solve Einstein's equations for a time-dependent cylindrical star perturbed slightly away from equilibrium. Since the equilibrium solution is static, we can assume that all perturbations have a time dependence $e^{i \omega t}$ and then solve for the quasinormal mode frequencies $\omega_{n}$. These frequencies have a discrete spectrum because of boundary conditions at infinity and at the center and surface of the star. This 
method directly treats the coupling of the fluid perturbations to the gravitational radiation. Unlike the $C$-energy method, it does not depend on the a priori knowledge of the stability of the system in some limit.

Recall that in the spherical case, one finds for radial perturbations that the Lagrangian fluid displacement $\xi$ obeys a second-order Sturm-Liouville eigenvalue equation with eigenvalues $\omega_{n}^{2}$, and that the perturbation in the gravitational field is tied to the fluid displacement [11]. In such a case, one can determine stability by solving for the fundamental eigenvalue $\omega_{0}^{2}$ : If $\omega_{0}^{2}>0$ then all radial modes are stable. If it is negative, the star is unstable.

In the cylindrical case, instead of a single eigenvalue equation one finds two coupled eigenvalue equations: One for the perturbation in the fluid, and another for the perturbation in the gravitational field. There are two equations instead of one because the field perturbation is no longer rigidly tied to the fluid displacement as it is in the spherical case-gravitational waves are possible in cylindrical symmetry. These gravitational waves cause an additional complication: In the spherical case, the solution in the vacuum outside the star is the Schwarzschild solution even in the presence of radial pulsations. However, in the cylindrical case, the solution outside of a radially pulsating star is not the Levi-Civita vacuum solution - it is instead a solution containing, in general, a superposition of incoming and outgoing gravitational waves. When diagnosing stability, one is interested in the case in which the only gravitational waves are those produced by the pulsations of the star, so one fixes the boundary condition at infinity so that only outgoing waves are present. This condition constrains $\omega_{n}$ to a discrete spectrum, and also introduces phase differences between the perturbed metric functions, so that the metric perturbations must be taken to be complex. In addition, the eigenvalues $\omega_{n}$ are themselves complex: The real part is the actual oscillation frequency and the imaginary part determines the stability of the star. If $\operatorname{Im}\left(\omega_{n}\right)>0$, the oscillations are damped in time because of energy loss to gravitational radiation, but if $\operatorname{Im}\left(\omega_{n}\right)<0$, the oscillation amplitude increases exponentially, and the star is therefore unstable.

\section{A. Perturbation equations}

When small radial perturbations from equilibrium are allowed, the metric is a function of $t$ as well as of $r$. The line element can be written in the form

$$
\begin{aligned}
d s^{2}= & -e^{2(v+\delta v)} d t^{2}+e^{2(\gamma-\psi+\delta \gamma-\delta \psi)} d r^{2} \\
& +e^{2(\psi+\delta \psi)} d z^{2}+r^{2} e^{-2(\psi+\delta \psi)} d \phi^{2}
\end{aligned}
$$

where $\delta v, \delta \gamma$, and $\delta \psi$ are the perturbations of the metric functions about equilibrium. Likewise, let $\delta \rho$ and $\delta p$ denote the deviations of $\rho$ and $p$ from equilibrium.

To zeroth order in all small quantities, Einstein's field equations give the equilibrium relations (10). To first order in these same quantities, Einstein's equations give, for a polytropic equation of state (see Appendix B),

$$
\begin{aligned}
& \xi^{\prime \prime} \Gamma p=\xi\left[X_{1}-B^{2} \omega^{2}(p+\rho)\right]+\xi^{\prime} X_{2}+\delta \psi X_{3}+\delta \psi^{\prime} X_{4}, \\
& -\delta \psi^{\prime \prime}=\xi Y_{1}+\xi^{\prime} Y_{2}+\delta \psi\left(B^{2} \omega^{2}+Y_{3}\right)+\delta \psi^{\prime} Y_{4},
\end{aligned}
$$

where primes denote differentiation with respect to $r$, and

$$
\begin{aligned}
& X_{1} \equiv A^{2} p^{2}\left(\frac{\rho}{n}-p\right) \\
& +\frac{A}{2 r}\left[\rho^{2}-\Gamma \rho p+\frac{p^{2}}{n}+y_{2}\left[\rho^{2}+\Gamma p^{2}+(1-2 \Gamma) p \rho\right]\right] \\
& +y_{3}\left[\frac{\rho+p}{r}(\Gamma-2)+y_{3}\left(\frac{\rho}{n}-p\right)\right] \text {, } \\
& Y_{1} \equiv \frac{A B}{2 r}\left[\rho^{\prime}-p^{\prime}+A\left[2 \rho^{2}-\rho p+(\Gamma-3) p^{2}\right]\right. \\
& \left.+\left[\rho-\frac{p}{n}\right]\left[\frac{1}{r}+y_{3}\right)\right] \text {, } \\
& X_{2} \equiv \Gamma\left[(\rho-p) y_{3}-p / r\right] \text {, } \\
& X_{3} \equiv B\left(y_{3}-A p\right)(\rho / n-p), \\
& \boldsymbol{X}_{4} \equiv \boldsymbol{B}(\rho-p / n) \text {, } \\
& Y_{2} \equiv(A B / 2 r)(\rho-p / n) \text {, } \\
& Y_{3} \equiv\left(B^{2} A / 2 r\right)[(3-\Gamma) p-\rho] \text {, } \\
& Y_{4} \equiv(p-\rho) A+1 / r \text {, } \\
& A \equiv 8 \pi r e^{2 \gamma-\psi}=\frac{8 \pi r e^{2 v}}{1-8 M}, \\
& B \equiv-(1-8 M)^{-1 / 2} \text {, } \\
& y_{2} \equiv B^{2}-1=\frac{1}{1-8 M}-1 \text {, } \\
& y_{3} \equiv y_{2} / 4 r \text {. }
\end{aligned}
$$

The two equations (20) and (21) are the coupled eigenvalue equations from which one determines the eigenfrequencies $\omega_{n}$. Since the functions $X_{n}$ and $Y_{n}$ appearing in Eqs. (22) depend only on equilibrium quantities, they can be treated as functions of $r$ alone. In addition, Eqs. (20) and $(21)$ reduce to a single equation in vacuum:

$$
\frac{\left(r \delta \psi^{\prime}\right)^{\prime}}{r}+B^{2} \omega^{2} \delta \psi=0 \text {. }
$$

This equation describes the radial dependence of gravitational waves outside the star, and its general solution is a superposition of Bessel functions.

\section{B. Complex search method}

Since Eqs. (20) and (21) are both second order, there will be four arbitrary constants that characterize each independent solution of the system. However, some of these degrees of freedom are removed by the physical constraint that the functions $\xi$ and $\delta \psi$ are finite everywhere: Because both equations contain terms proportional to $1 / r$ or $1 / r^{2}$, this constraint requires the follow- 
ing boundary conditions at the origin:

$$
\xi=\delta \psi^{\prime}=0 \text { at } r=0 \text {. }
$$

Therefore there are two independent solutions to Eqs. (20) and (21) that are regular at the origin: these will be called "inner" solutions, and are parametrized by the values of $\xi^{\prime}$ and $\delta \psi$ at $r=0$. Likewise, since $p$ vanishes at the surface of the star, the requirement that $\xi^{\prime \prime}$ be finite results in a boundary condition at $r=r_{s}$ :

$$
\begin{aligned}
\xi[- & \left.B^{2} \omega^{2}+y_{3}\left(\frac{\Gamma-2}{r}+\frac{y_{3}}{n}\right)\right] \\
& +\xi^{\prime} \Gamma y_{3}+\delta \psi^{\prime} B+\delta \psi \frac{B y_{3}}{n}=0 \text { at } r=r_{s} .
\end{aligned}
$$

There are thus three independent solutions to Eqs. (20) and (21) that are regular at the surface of the star: these will be called "outer" solutions, and are parametrized by the values of any three of the four quantities $\xi, \delta \psi, \delta \psi^{\prime}$, and $\xi^{\prime}$ at $r=r_{s}$ [the fourth quantity is related to the other three by Eq. (25)]. Note that there is not yet any requirement on the form of the gravitational waves outside of the star.

For any complex value of $\omega$, one can find a solution that is regular everywhere; i.e., some linear combination of the "inner" solutions will be equal to a linear combination of the "outer" solutions, as long as one still allows an arbitrary mixture of incoming and outgoing gravitational waves in the vacuum. However, if one imposes the additional constraint that there are only outgoing waves at infinity, the continuity of the metric at the surface of the cylinder imposes the additional boundary condition

$$
\frac{\delta \psi^{\prime}\left(r_{s}\right)}{\delta \psi\left(r_{s}\right)}=\omega B_{s} \frac{H_{1}^{(2)}\left(r_{s}^{*}\right)}{H_{0}^{(2)}\left(r_{s}^{*}\right)},
$$

where $H$ denotes a Hankel function and $r_{s}^{*} \equiv \omega\left|B_{s}\right| r_{s}$. This condition reduces the number of independent solutions at the surface of the cylinder from three to two, and as a result, a solution for the entire cylinder exists only for discrete values of $\omega$; i.e., for discrete eigenvalues $\omega_{n}$ there exists a linear combination of the "inner" two solutions that is equal to a linear combination of the "outer" two solutions. Since the Hankel functions are complex, this condition also introduces phase differences between the various perturbed quantities, and therefore requires all of these quantities to be taken as complex.

One can determine whether or not a chosen complex value of $\omega$ is an eigenvalue of Eqs. (20) and (21) by a shooting method. First numerically integrate the two "inner" solutions from the center of the cylinder to some joining point $r_{j}<r_{s}$, thus obtaining two sets of values for $\xi\left(r_{j}\right), \xi^{\prime}\left(r_{j}\right), \delta \psi\left(r_{j}\right)$, and $\delta \psi^{\prime}\left(r_{j}\right)$. Then numerically integrate the two "outer" solutions from $r=r_{s}$ to $r=r_{j}$, thus obtaining two more sets of values for $\xi\left(r_{j}\right), \xi^{\prime}\left(r_{j}\right)$, $\delta \psi\left(r_{j}\right)$, and $\delta \psi^{\prime}\left(r_{j}\right)$. Finally, construct a complex $4 \times 4$ matrix from these four sets of values and evaluate its determinant $D$. If there exists a solution with the chosen value of $\omega$ that is regular everywhere, i.e., if $\omega$ is equal to an eigenvalue $\omega_{n}$, then the four sets of values of $\xi\left(r_{j}\right)$, $\xi^{\prime}\left(r_{j}\right), \delta \psi\left(r_{j}\right)$, and $\delta \psi^{\prime}\left(r_{j}\right)$ are linearly dependent, and hence $D$ vanishes.

Therefore, the problem of finding the eigenvalues $\omega_{n}$ reduces to one of finding the roots of $D(\omega)$. This task is not completely trivial since $D(\omega)$ is a complex function of a complex variable, but it can be accomplished using Newton's method. However, since Newton's method is in general only successful if one has a fairly accurate initial guess for the position of the root, it would be helpful to have an alternative method for finding eigenvalues, such as the one provided by the resonance method.

\section{Resonance method}

Many of the practical difficulties associated with the direct method of finding the eigenvalues of Eqs. (20) and (21) arise because both the eigenvalues $\omega_{n}$ and the eigenfunctions $\xi$ and $\delta \psi$ are complex. However, if the imaginary part of the desired eigenvalue is much less than its real part, it turns out that one may determine both the real and imaginary parts of $\omega_{n}$ by only examining the solutions of Eqs. (20) and (21) for which $\xi, \delta \psi$, and $\omega$ are real, as will be shown below.

Imagine that one is interested in a complex solution of Eqs. (20) and (21), and one does not impose the outgoingwave boundary condition (26). In this case, a solution exists for any complex value of $\omega$, and in the vacuum region outside the star, this solution is simply the solution of Eq. (23), which can be written in the form

$$
\delta \psi=C_{\mathrm{in}} H_{0}^{(1)}\left(r^{*}\right)+C_{\mathrm{out}} H_{0}^{(2)}\left(r^{*}\right),
$$

where $r^{*} \equiv \omega\left|B_{s}\right| r, H$ denotes a Hankel function, and $C_{\text {in }}$ and $C_{\text {out }}$ are the complex amplitudes of incoming and outgoing gravitational waves, which depend on the parameter $\omega$. Since the eigenvalues $\omega_{n}$ are simply the solutions for which the amplitude $C_{\text {in }}$ vanishes, the problem of finding the eigenvalues can be recast into one of finding the poles of the function $\left|C_{\text {in }}(\omega)\right|^{-1}$.

Let the desired complex eigenvalue be denoted by $\omega_{n}=\omega_{r}+i \omega_{i}$. If one expands $C_{\text {in }}(\omega)$ about $\omega_{n}$, one obtains

$$
\begin{aligned}
& C_{\text {in }}(\omega)=C_{\text {in }}\left(\omega_{n}\right)\left(\omega-\omega_{r}-i \omega_{i}\right) \\
& \text { so }\left|C_{\text {in }}(\omega)\right|^{-2} \propto \frac{1}{\left(\omega-\omega_{r}\right)^{2}+\omega_{i}^{2}},
\end{aligned}
$$

which is the Breit-Wigner expression that appears in the theory of resonance scattering in quantum mechanics. An enormous simplification arises from the fact that if $\left|\omega_{i}\right| \ll\left|\omega_{r}\right|$, the above Taylor expansion remains valid for $\omega$ on the real axis near $\omega_{r}$. Therefore, instead of searching the entire complex plane for poles, in the case $\left|\omega_{i}\right| \ll\left|\omega_{r}\right|$ one may merely search the real axis for maxima: According to Eq. (28), the position of each maximum gives the real part of one of the eigenvalues $\omega_{r}$, and the width of that maximum gives the corresponding imaginary part $\left|\omega_{i}\right|$.

This method is not yet sufficient to diagnose stability, for it does not give the sign of $\omega_{i}$. However, as shown in Appendix A [12], if one writes the exterior solution for 
real $\omega$ in the form

$$
\delta \psi=\alpha J_{0}\left(r^{*}\right)-\beta N_{0}\left(r^{*}\right),
$$

where $\alpha$ and $\beta$ are considered to be functions of $\omega$, then the imaginary part of $\omega_{n}$ is given by

$$
\omega_{i}=\frac{\alpha}{\partial \beta / \partial \omega}=\frac{-\beta}{\partial \alpha / \partial \omega},
$$

evaluated at $\omega=\omega_{r}$. Note also from Eq. (29) that the amplitude of incoming gravitational radiation for real $\omega$ is given by

$$
\left|C_{\mathrm{in}}(\omega)\right|^{-1} \propto\left(\alpha^{2}+\beta^{2}\right)^{-1 / 2} .
$$

The procedure for finding eigenvalues by the resonance method is the following: For a chosen real value of $\omega$, fix real values of $\xi^{\prime}$ and $\delta \psi$ at the origin, and integrate the two "inner" solutions of Eqs. (20) and (21) from the origin to a joining point $\boldsymbol{r}_{j}$, thus obtaining two sets of real values for $\xi\left(r_{j}\right), \xi^{\prime}\left(r_{j}\right), \delta \psi\left(r_{j}\right)$, and $\delta \psi^{\prime}\left(r_{j}\right)$. Then, allowing an arbitrary mixture of incoming and outgoing waves, integrate the three "outer" solutions from $r=r_{s}$ to $r=r_{j}$, starting with fixed real values of $\xi, \xi^{\prime}, \delta \psi$, and $\delta \psi^{\prime}$ that satisfy the boundary condition (25), thus obtaining three more sets of real values for $\xi\left(r_{j}\right), \xi^{\prime}\left(r_{j}\right), \delta \psi\left(r_{j}\right)$, and $\delta \psi^{\prime}\left(r_{j}\right)$. Finally, construct a solution which is regular everywhere by finding the linear combination of the three "outer" solutions which is equal to a linear combination of the two "inner" solutions. This solution is unique up to an arbitrary normalization, which we chose such that $\xi^{\prime 2}+\delta \psi^{2}=1$ at the center of the cylinder. From this solution, determine the amplitudes $\alpha$ and $\beta$ by matching the surface values of $\delta \psi$ and $\delta \psi^{\prime}$ to Eq. (29), and then repeat the entire procedure for different real values of $\omega$. The real parts of the eigenvalues $\omega_{n}$ are then equal to the maxima of the function

$$
\left[\alpha(\omega)^{2}+\beta(\omega)^{2}\right]^{-1 / 2},
$$

and the imaginary parts of the eigenvalues are found from Eq. (30).

One difficulty with the resonance method is that the functions $\alpha(\omega)$ and $\beta(\omega)$ can vary quite rapidly with $\omega$ near the eigenvalues, and therefore computing their derivatives [which are required to determine $\omega_{i}$ from Eq. (30)] involves evaluating these functions for values of $\omega$ that differ by an extremely small amount. This requires a high degree of numerical precision, sometimes exceeding the 16-digit limit of double-precision arithmetic. On the other hand, if $\alpha(\omega)$ and $\beta(\omega)$ do not vary rapidly with $\omega$ near the eigenvalues, then $\omega_{i}$ is no longer much less than $\omega_{r}$ and the method fails.

\section{NONDIMENSIONALIZED FIELD EQUATIONS AND ULTRARELATIVISTIC SCALING}

For numerical work, it is useful to obtain a dimensionless parametrization of the field equations. Since the constant $K$ appearing in the polytropic equation of state (12) has dimensions of (length $)^{2 / n}$, we can write

$$
\begin{aligned}
& p \equiv K^{-n} \widetilde{q}^{n+1}, \\
& \rho \equiv K^{-n} \widetilde{\rho}, \\
& r \equiv K^{n / 2} \widetilde{r}, \\
& \xi \equiv K^{n / 2} \widetilde{\xi}, \\
& \omega \equiv K^{-n / 2} \widetilde{\omega},
\end{aligned}
$$

where the quantities with tildes are dimensionless. In terms of these variables, the polytropic equation of state (12) takes the form

$$
\widetilde{\rho}=\widetilde{q}^{n}(1+n \widetilde{q}) \text {. }
$$

The quantity $\widetilde{q}_{c}$, which is equal to the ratio of the central pressure to the central rest energy density, can be thought of as a "relativity parameter": For $\widetilde{q}_{c} \gg 1$ the matter is extremely relativistic, and for $\widetilde{q}_{c}<<1$ it is nonrelativistic.

In terms of these dimensionless variables, the equilibrium equations (10) become

$$
\begin{aligned}
& M^{\prime}=2 \pi \widetilde{r} \widetilde{q}^{n}[1+\widetilde{q}(n-1)] e^{2 v}, \\
& \psi^{\prime}=(1 / 2 \widetilde{r})\left[1-(1-8 M)^{-1 / 2}\right], \\
& v^{\prime}=\frac{2}{\widetilde{r}(1-8 M)}\left(M+4 \pi \widetilde{r}^{2} e^{2 v} \widetilde{q}^{n+1}\right), \\
& \widetilde{q}^{\prime}=-v^{\prime}\left(\widetilde{q}+\frac{1}{n \Gamma}\right],
\end{aligned}
$$

subject to the boundary conditions

$$
\begin{array}{r}
M=M^{\prime}=v=v^{\prime}=\psi=\psi^{\prime}=\widetilde{q}^{\prime}=0, \\
\quad \widetilde{q}=\widetilde{q}_{c}, \text { at } \widetilde{r}=0,
\end{array}
$$

where primes denote derivatives with respect to the new dimensionless variable $\widetilde{r}$. Likewise, the perturbation equations (20) and (21) become

$$
\begin{array}{r}
\frac{\left(\widetilde{r} \delta \psi^{\prime}\right)^{\prime}}{\widetilde{r}}-\delta \psi^{\prime} \widetilde{q}^{n}[1+\widetilde{q}(n-1)] A+B^{2} \delta \psi\left[\widetilde{\omega}^{2}-\frac{A \widetilde{q}^{n}}{2 \widetilde{r}}[1+\widetilde{q}(n+\Gamma-3)]+\widetilde{\xi}^{\prime} \frac{A B \widetilde{q}^{n}}{2 \widetilde{r}}\left[1+\widetilde{q}\left[n-\frac{1}{n}\right]\right]\right. \\
+\widetilde{\xi} \frac{A B}{2 \widetilde{r}}\left[A \widetilde{q}^{2 n}\left[2-\frac{1}{\Gamma}+\widetilde{q}\left[2 n+\widetilde{q}\left(n^{2}-n+\Gamma-2\right)\right]\right)+\frac{\widetilde{q}^{n}}{\widetilde{r}}\left[1+\widetilde{q}\left[n-\frac{1}{n}\right]\right]\right. \\
+y_{3} \widetilde{q}^{n-1}\left(\frac{-1}{\Gamma}+\widetilde{q}(1-n)[2+\widetilde{q} \Gamma(n-1)]\right]=0,
\end{array}
$$




$$
\begin{aligned}
\widetilde{\xi}^{\prime \prime} \Gamma= & \frac{1}{\widetilde{q}}\left\{\widetilde{\xi}\left[-B^{2} \widetilde{\omega}^{2}+y_{3}\left(\frac{\Gamma-2}{\widetilde{r}}+\frac{y_{3}}{n}\right]\right)+\widetilde{\xi}^{\prime} \Gamma y_{3}+\delta \psi^{\prime} B+\delta \psi B \frac{y_{3}}{n}\right\}+\widetilde{\xi} \Gamma\left[-B^{2} \widetilde{\omega}^{2} n+\frac{1}{\widetilde{r}^{2}}+\frac{n y_{3}}{\widetilde{r}}(\Gamma-2)\right] \\
& +\widetilde{\xi}^{\prime} \Gamma\left[(n-1) y_{3}-\frac{1}{\widetilde{r}}\right]+\delta \psi^{\prime} B\left[n-\frac{1}{n}\right]+\frac{A \widetilde{q}^{n-1} \xi}{2 \widetilde{r}}\left(B^{2}+\widetilde{q}\left\{2 n-\Gamma+y_{2}[2(n-\Gamma)+1]+B^{2} \widetilde{q} \Gamma(n-1)^{2}\right\}\right) \\
& +\frac{A \widetilde{q}^{n}}{n}\left(-\delta \psi B+\widetilde{\xi} A \widetilde{q}^{n+1}\right),
\end{aligned}
$$

where

$$
\begin{aligned}
& A \equiv 8 \pi \widetilde{r} e^{2 \gamma-\psi}=8 \pi \widetilde{r} e^{2 v} /(1-8 M), \\
& B \equiv-(1-8 M)^{-1 / 2}, \\
& y_{2} \equiv B^{2}-1=[1 /(1-8 M)]-1 \\
& y_{3} \equiv y_{2} / 4 \widetilde{r} .
\end{aligned}
$$

The variables $\widetilde{\xi}$ and $\delta \psi$ must satisfy the boundary conditions

$$
\begin{aligned}
& \widetilde{\xi}=\delta \psi^{\prime}=0 \text { at } \widetilde{r}=0, \\
& \widetilde{\xi}\left[-B^{2} \widetilde{\omega}^{2}+y_{3}\left[\frac{\Gamma-2}{\widetilde{r}}+\frac{y_{3}}{n}\right)\right] \\
& \quad+\widetilde{\xi}^{\prime} \Gamma y_{3}+\delta \psi^{\prime} B+\delta \psi \frac{B y_{3}}{n}=0 \text { at } \widetilde{r}=\widetilde{r}_{s} .
\end{aligned}
$$

In addition, if one imposes the condition that only outgoing waves must exist at infinity (as in the complex search method), then one must have

$$
\frac{\delta \psi^{\prime}\left(\widetilde{r}_{s}\right)}{\delta \psi\left(\widetilde{r}_{s}\right)}=\widetilde{\omega} B_{s} \frac{H_{1}^{(2)}\left(r_{s}^{*}\right)}{H_{0}^{(2)}\left(r_{s}^{*}\right)},
$$

where

$$
r_{s}^{*} \equiv \omega\left|B_{s}\right| r_{s} \equiv \widetilde{\omega}\left|B_{s}\right| \widetilde{r}_{s}
$$

and $H$ is a Hankel function.

\section{Ultrarelativistic limit}

Notice that the magnitudes of the dimensionless variables $\widetilde{\xi}, \widetilde{r}, \widetilde{\omega}$, and $\widetilde{q}$ depend on the relativity parameter $\widetilde{q}_{c}$. Because of this, the above equations do not take on a particularly simple form in the ultrarelativistic limit. However, if one defines new dimensionless variables by "factoring out" various powers of $\widetilde{q}_{c}$, then the above equations, expressed in terms of these new variables, become independent of $\widetilde{q}_{c}$ in the ultrarelativistic limit. To do this, let

$$
\begin{aligned}
& \widetilde{q} \equiv \widetilde{q}_{c}, \\
& \widetilde{r} \equiv \overline{r q}_{c}^{-(n+1) / 2}, \\
& \widetilde{\xi} \equiv \bar{\xi} \widetilde{q}_{c}^{-(n+1) / 2}, \\
& \widetilde{\omega} \equiv \bar{\omega} \widetilde{q}_{c}^{(n+1) / 2},
\end{aligned}
$$

and let the metric functions remain unchanged. If one defines $S \equiv 1 / \widetilde{q}_{c}$, the equilibrium and perturbation equations become

$$
\begin{aligned}
& M^{\prime}=2 \pi \bar{r} \bar{q}^{n}[S+\bar{q}(n-1)] e^{2 v}, \\
& \psi^{\prime}=(1 / 2 \bar{r})\left[1-(1-8 M)^{-1 / 2}\right], \\
& v^{\prime}=[2 / \bar{r}(1-8 M)]\left(M+4 \pi \bar{r}^{2} e^{2 v} \bar{q}^{n+1}\right), \\
& \bar{q}^{\prime}=-v^{\prime}(\bar{q}+S / n \Gamma),
\end{aligned}
$$

subject to the boundary conditions

$M=M^{\prime}=v=v^{\prime}=\psi=\psi^{\prime}=\bar{q}^{\prime}=0, \bar{q}=1$ at $\bar{r}=0$,

where primes now denote differentiation with respect to $\bar{r}$. The perturbation equations become

$$
\begin{array}{r}
\left.\left.\left.\frac{\left(\bar{r} \delta \psi^{\prime}\right)^{\prime}}{\bar{r}}-\delta \psi^{\prime} \bar{q}^{n}[S+\bar{q}(n-1)] A+B^{2} \delta \psi\left[\bar{\omega}^{2}-\frac{A \bar{q}^{n}}{2 \bar{r}}[S+\bar{q}(n+\Gamma-3)]\right]+\bar{\xi}^{\prime} \frac{A B \bar{q}^{n}}{2 \bar{r}}[S+\bar{q}] n-\frac{1}{n}\right]\right]\right] \\
+\bar{\xi} \frac{A B}{2 \bar{r}}\left[A \overline { q } ^ { 2 n } \left[2 S^{2}-\frac{S^{2}}{\Gamma}+\bar{q}\left[2 n S+\bar{q}\left(n^{2}-n+\Gamma-2\right)\right]+\frac{\bar{q}^{n}}{\bar{r}}\left[S+\bar{q}\left[n-\frac{1}{n}\right]\right]\right.\right. \\
+y_{3} \bar{q}^{n-1}\left[\frac{-S^{2}}{\Gamma}+\bar{q}(1-n)[2 S+\bar{q} \Gamma(n-1)]\right]=0,
\end{array}
$$




$$
\begin{aligned}
\bar{\xi}^{\prime \prime} \Gamma= & \frac{S}{\bar{q}}\left\{\bar{\xi}\left[-B^{2} \bar{\omega}^{2}+y_{3}\left[\frac{\Gamma-2}{\bar{r}}+\frac{y_{3}}{n}\right]\right]+\bar{\xi}^{\prime} \Gamma y_{3}+\delta \psi^{\prime} B+\delta \psi B \frac{y_{3}}{n}\right\}+\bar{\xi} \Gamma\left[-B^{2} \bar{\omega}^{2} n+\frac{1}{\bar{r}^{2}}+\frac{n y_{3}}{\bar{r}}(\Gamma-2)\right] \\
& +\bar{\xi}^{\prime} \Gamma\left[(n-1) y_{3}-\frac{1}{\bar{r}}\right]+\delta \psi^{\prime} B\left[n-\frac{1}{n}\right] \\
& +\frac{A \bar{q}^{n-1 \bar{\xi}}}{2 \bar{r}}\left(B^{2} S^{2}+\bar{q}\left\{2 n S-\Gamma S+S y_{2}[2(n-\Gamma)+1]+B^{2} \bar{q} \Gamma(n-1)^{2}\right\}\right)+\frac{A S \bar{q}^{n}}{n}\left(-\delta \psi B+\bar{\xi} A \bar{q}^{n+1}\right),
\end{aligned}
$$

where

$$
\begin{aligned}
& A \equiv 8 \pi \bar{r} e^{2 \gamma-\psi}=8 \pi \bar{r} e^{2 v} /(1-8 M), \\
& B \equiv-(1-8 M)^{-1 / 2} \\
& y_{2} \equiv B^{2}-1=1 /(1-8 M)-1, \\
& y_{3} \equiv y_{2} / 4 \bar{r} .
\end{aligned}
$$

The boundary conditions (39) can be written

$$
\begin{aligned}
& \bar{\xi}=\delta \psi^{\prime}=0 \text { at } \bar{r}=0, \\
& \bar{\xi}\left[-B^{2} \bar{\omega}^{2}+y_{3}\left[\frac{\Gamma-2}{\bar{r}}+\frac{y_{3}}{n}\right]\right]+\bar{\xi}^{\prime} \Gamma y_{3}+\delta \psi^{\prime} B \\
& +\delta \psi\left(B y_{3} / n\right)=0 \text { at } \bar{r}=\bar{r}_{s},
\end{aligned}
$$

and the outgoing-wave boundary condition (40) remains the same except tildes are replaced by bars.

In these equations, all the information about the degree of relativity of the system is contained within the parameter $S$. In the ultrarelativistic limit, $S$ becomes very small and can therefore be neglected in most of the star; the only place where $S$ cannot be neglected is in the region close to the surface of the cylinder where $\bar{q} \lesssim S$. However, in this surface region, the Lane-Emden variable $\bar{q}$, and hence the energy density, is negligibly small, so the variables $v, \psi, M$, and $\delta \psi$ vary with $\bar{r}$ as if they were in vacuum. Consequently, the quantities $v, \psi, M$, and $\delta \psi$, when expressed as functions of the dimensionless variable $\bar{r}$, are independent of $S$ in the ultrarelativistic limit, both in the interior and exterior of the cylinder.

The nondimensional radius of the cylinder $\bar{r}_{s}$, defined as the value of $\bar{r}$ at which $\bar{q}$ vanishes, increases as $S$ becomes small. For the asymptotic solution $(S=0), \bar{q}$ falls off exponentially at large $\bar{r}$, so $\bar{r}_{s}$ is infinite. However, if one defines $\bar{r}_{\epsilon}$ as the value of $\bar{r}$ at which $\bar{q}$ becomes less than some small number $\epsilon>0$, then $\bar{r}_{\epsilon}$ is independent of $S$ for sufficiently small $S$. The quantity $\bar{r}_{\epsilon}$ can be thought of as the effective nondimensional radius of the cylinder since the region outside of $\bar{r}_{\epsilon}$ is very nearly vacuum. One can similarly define the quantity

$$
R_{\epsilon} \equiv\left(\bar{r}_{\epsilon} / \bar{r}_{s}\right) R_{s},
$$

which has dimensions of length, as the effective circumferential radius of the cylinder.

Because the equilibrium equations and perturbation equations, when expressed in terms of barred variables, become effectively independent of $\widetilde{q}_{c}(=1 / S)$ in the ultrarelativistic limit, one finds that the physical variables scale with $\widetilde{q}_{c}$ according to simple power laws: From Eqs. (32) and (41) one obtains

$$
\begin{aligned}
& R_{\epsilon} \propto \widetilde{q}_{c}^{-(n+1) / 2}, \\
& \omega^{2} \propto \rho_{c} \propto p_{c} \propto \widetilde{q}_{c}^{n+1}, \\
& \psi, v, M, \delta \psi \text { independent of } \widetilde{q}_{c} .
\end{aligned}
$$

Furthermore, one can insert $R_{\epsilon}$ instead of $R_{s}$ into Eq. (18) to obtain

$$
\gamma_{b} \propto \text { const }+\ln \left(\widetilde{q}_{c}\right) .
$$

The last step is valid because Eq. (18) was derived by integrating the vacuum equilibrium equations starting at the surface of the cylinder; using $R_{\epsilon}$ rather than $R_{s}$ is equivalent to integrating the same vacuum equations, but starting inside the cylinder at a point where the pressure is arbitrarily small but nonzero.

\section{RESULTS}

\section{A. C-energy method}

We constructed equilibrium models for several values of $\Gamma$ over a wide range of central densities $\rho_{c}$. A typical model with $\Gamma=1.5$ is shown in Fig. 1 . The metric
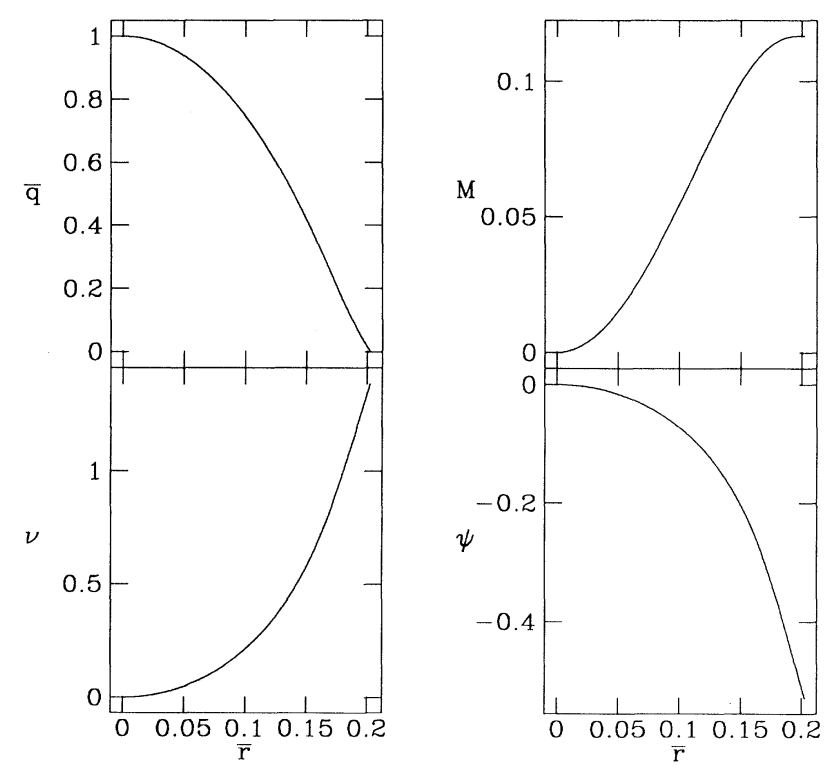

FIG. 1. Equilibrium model of a $\Gamma=1.5(n=2)$ cylindrical polytrope with $\widetilde{q}_{c}=1$. 
coefficient $v$ at the surface of the cylinder is of order unity in this example, indicating that the cylinder is quite relativistic-photons emitted from the axis are gravitationally redshifted by about a factor of 4 before they reach the surface. The function $\psi(r)$ is monotonically decreasing, so the coordinate separation between the end points of a meter stick oriented in the $z$ direction diverges as it is moved away from the axis. In addition, the proper circumference of the cylinder increases faster than $2 \pi r$ as one moves out from the axis, and the proper radius is greater than the coordinate radius $r$.

According to the scaling laws (49), the structure of an equilibrium cylinder in the dimensionless units defined in Eq. (41) should be independent of $\rho_{c}$ in the ultrarelativistic limit. This agrees well with our numerical results, as illustrated in Fig. 2: Solutions with finite values of $\widetilde{q}_{c}$ converge to an asymptotic solution as $\widetilde{q}_{c} \rightarrow \infty$. The pressure and energy density of this asymptotic solution drop off exponentially at large distances; hence, the true nondimensional radius $\bar{r}_{s}$ is infinite, whereas the effective nondimensional radius $\bar{r}_{\epsilon}$ is finite. For $\bar{r}>\bar{r}_{\epsilon}$, the pressure and energy density may be neglected.

Figures 3-5 show the $C$-energy parameter $\gamma_{b}$ and the Levi-Civita mass parameter $k$ plotted as a function of central energy density for three values of $\Gamma$. Also shown are the circumferential radius $R_{s}$ of the configuration and the surface values of the metric coefficients $v, \psi$, and $m$. The redshift factor $e^{v_{s}}=\sqrt{g_{00}}$ is a good indication of whether a particular configuration is relativistic: this fac-
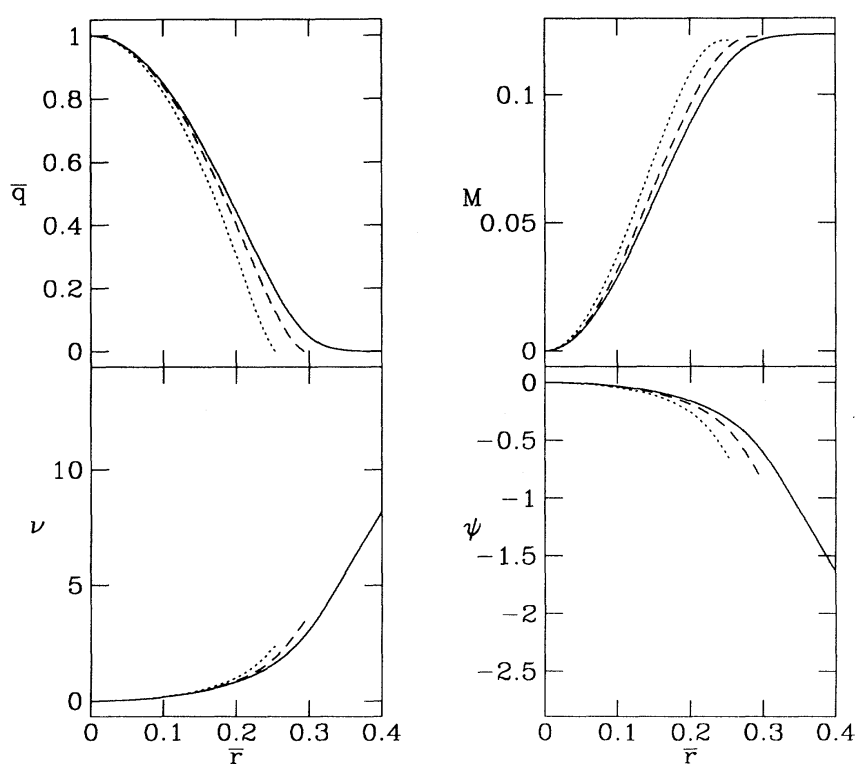

FIG. 2. Metric coefficients and nondimensional pressure for $\Gamma=1.5$ equilibrium cylinders with different relativity parameters $\widetilde{q}_{c}$ and nondimensional radii $\bar{r}_{s}$. The solid curves correspond to the asymptotic solution $\left(\widetilde{q}_{c}, \bar{r}_{s} \rightarrow \infty\right)$, and the dotted and dashed curves correspond to solutions with $\widetilde{q}_{c}=\sqrt{10}$ $\left(\bar{r}_{s} \sim 0.25\right)$ and $\widetilde{q}_{c}=10\left(\bar{r}_{s} \sim 0.3\right)$, respectively. On this plot, curves with $\widetilde{q}_{c} \gtrsim 100$ would coincide with the asymptotic solution.
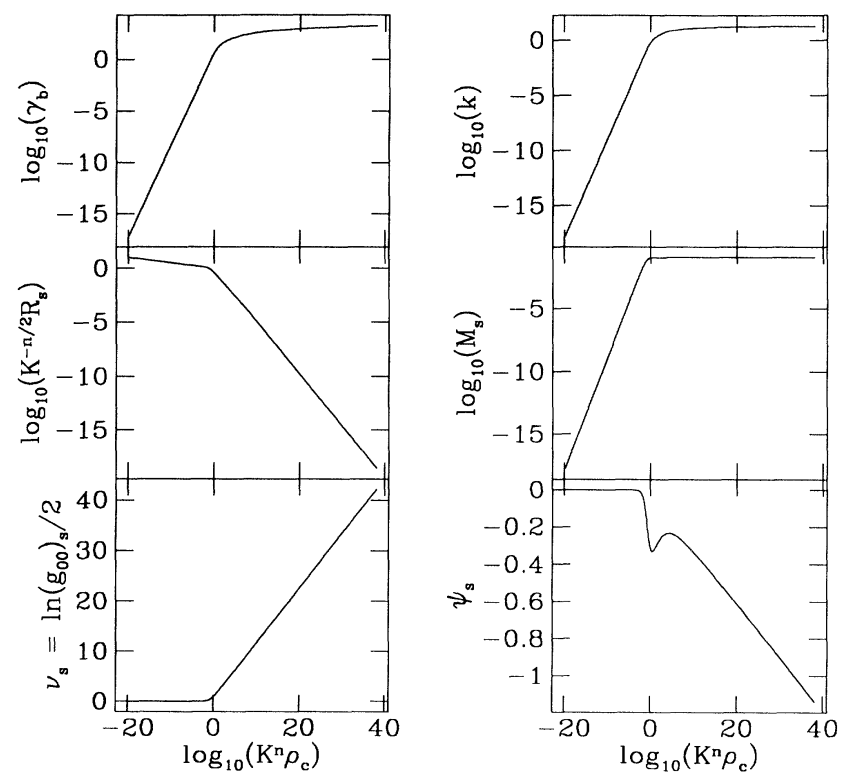

FIG. 3. Equilibrium sequences for $\Gamma=1.9(n=1.1)$. Note the logarithmic behavior of $\gamma_{b}\left(\rho_{c}\right)$ for large $\rho_{c}$. Also notice the large redshifts for large values of $\rho_{c}$.

tor is much smaller than unity for small $\rho_{c}$, and significantly larger than unity for large $\rho_{c}$, indicating that the plots encompass both the Newtonian and ultrarelativistic limits.

In the nonrelativistic limit, $M_{s}, k$, and $\gamma_{b}$ all reduce to the Newtonian rest mass per unit length of the cylinder. This is reflected in Figs. $3-5$ by the fact that $M_{s}, k$, and $\gamma_{b}$ are equal for small $\rho_{c}$. In the ultrarelativistic limit, $\gamma_{b}\left(\rho_{c}\right)$ behaves logarithmically, in agreement with the
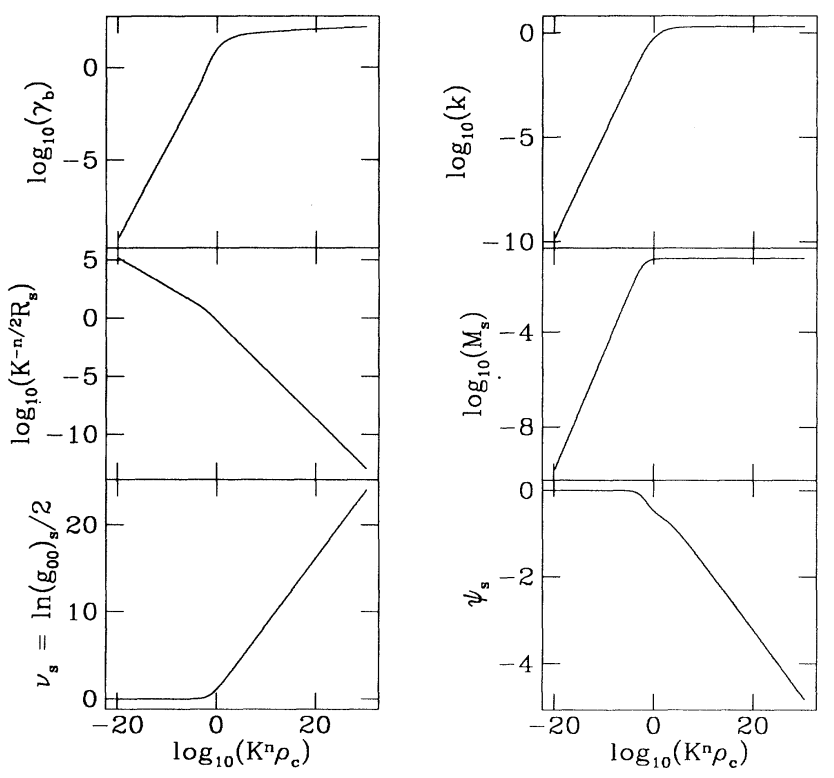

FIG. 4. Equilibrium sequences for $\Gamma=1.5(n=2)$. 

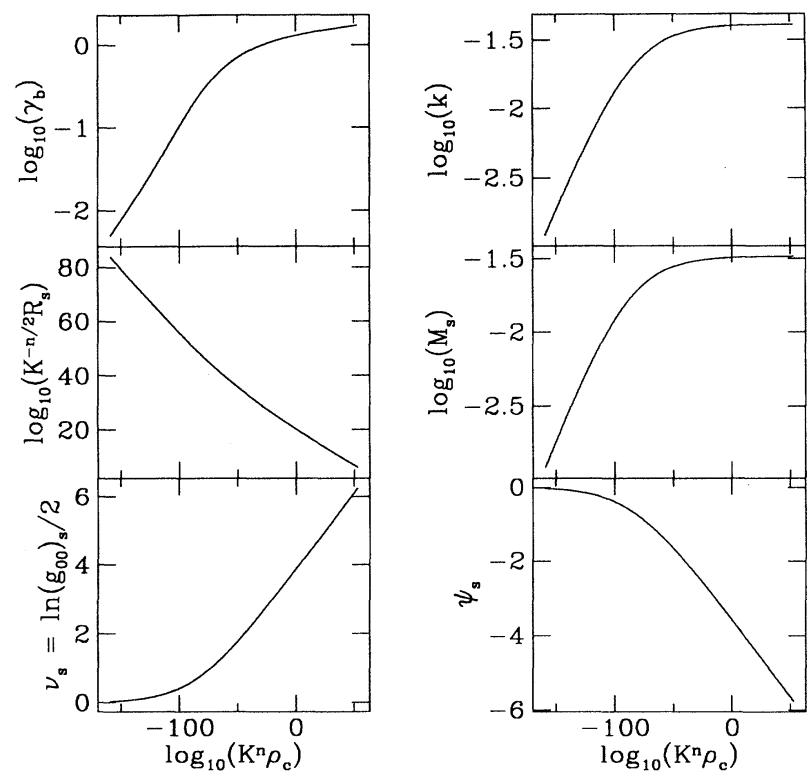

FIG. 5. Equilibrium sequences for $\Gamma=1.02(n=50)$.

scaling law (50). This can also be seen from Figs. 3-5, but is shown more clearly in Fig. 6, in which $\gamma_{b}$ is plotted on a linear axis.

In all cases considered, the slopes of both $\gamma_{b}\left(\rho_{c}\right)$ and $k\left(\rho_{c}\right)$ are positive for all $\rho_{c}$, indicating by property 4 that there is no radial acoustic mode that changes stability as a function of $\rho_{c}$. In addition, the logarithmic increase of $\gamma_{b}\left(\rho_{c}\right)$ for asymptotically large $\rho_{c}$ demonstrates that no radial acoustic mode changes stability as $\rho_{c}$ tends towards infinity. If all equilibrium cylindrical polytropes

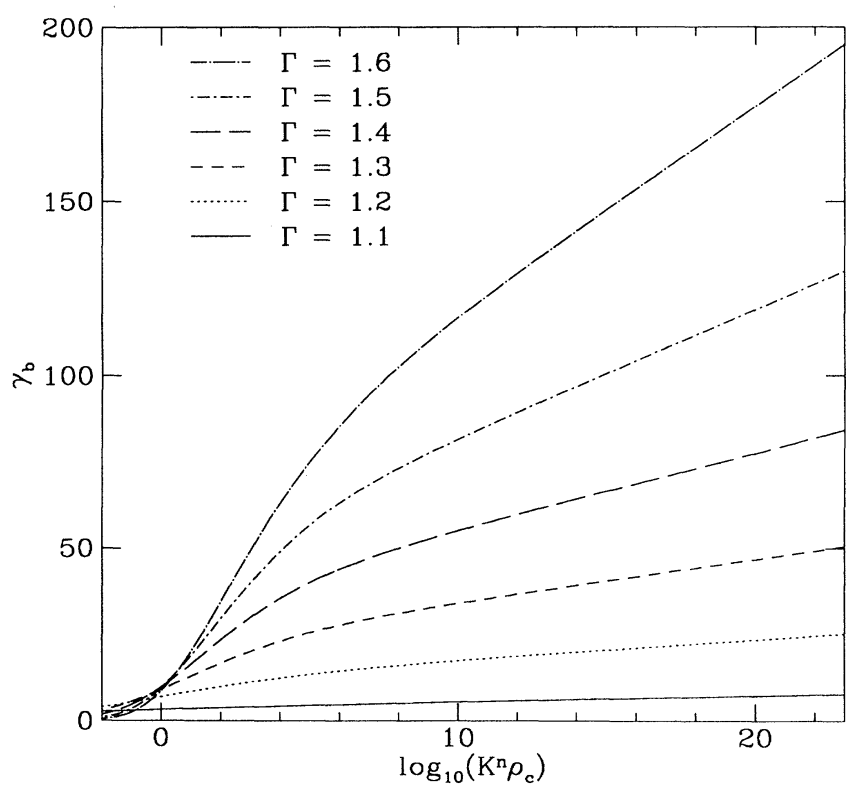

FIG. 6. Logarithmic behavior of $\gamma_{b}$ in the extremely relativistic limit, shown for several values of $\Gamma$.

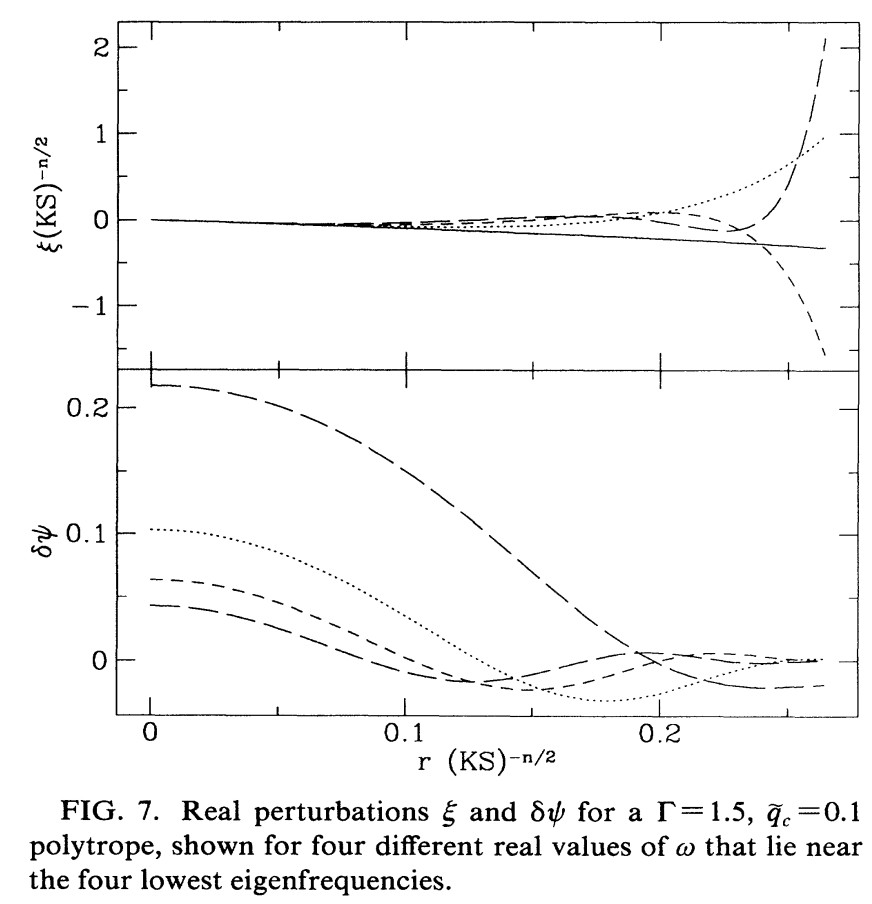

FIG. 7. Real perturbations $\xi$ and $\delta \psi$ for a $\Gamma=1.5, \widetilde{q}_{c}=0.1$ polytrope, shown for four different real values of $\omega$ that lie near the four lowest eigenfrequencies.

(with physically meaningful values of $\Gamma$ ) are stable in the nonrelativistic limit, this result implies that all relativistic cylindrical polytropes are stable as well. This is in sharp contrast to spherical systems, in which sufficiently large central pressure drives an instability that results in a collapse to a Schwarzschild black hole.

However, even though we know that all Newtonian cylindrical polytropes are stable with respect to radial perturbations, this does not guarantee that all cylindrical polytropes are stable in the nonrelativistic limit. The difference between the Newtonian case and the nonrelativistic limit is that the former does not allow any gravitational radiation: It is possible that a stable Newtonian cylinder becomes unstable when one introduces an infinitesimal amount of gravitational waves. If this were true, then the above analysis would show that relativistic cylindrical polytropes are actually unstable. In order to ensure that this is not the case, one must have an independent method of determining whether a particular configuration is stable. Explicitly calculating the quasinormal mode eigenfrequencies associated with radial perturbations is a good method for this purpose, since it does not require any knowledge of stability in the nonrelativistic limit.

\section{B. Complex eigenfrequencies}

We first determined the eigenfrequencies $\omega_{n}$ by the resonance method for several different values of $\Gamma$ and $\rho_{c}$. An example of the real nondimensional perturbation functions obtained from the numerical integration is shown in Fig. 7, and a typical plot of the wave amplitudes $\alpha$ and $\beta$ defined in Eq. (29) is illustrated in Fig. 8. Notice that at certain values of $\omega$, both $\alpha$ and $\beta$ pass close to zero-the values at which this occurs are the maxima of 


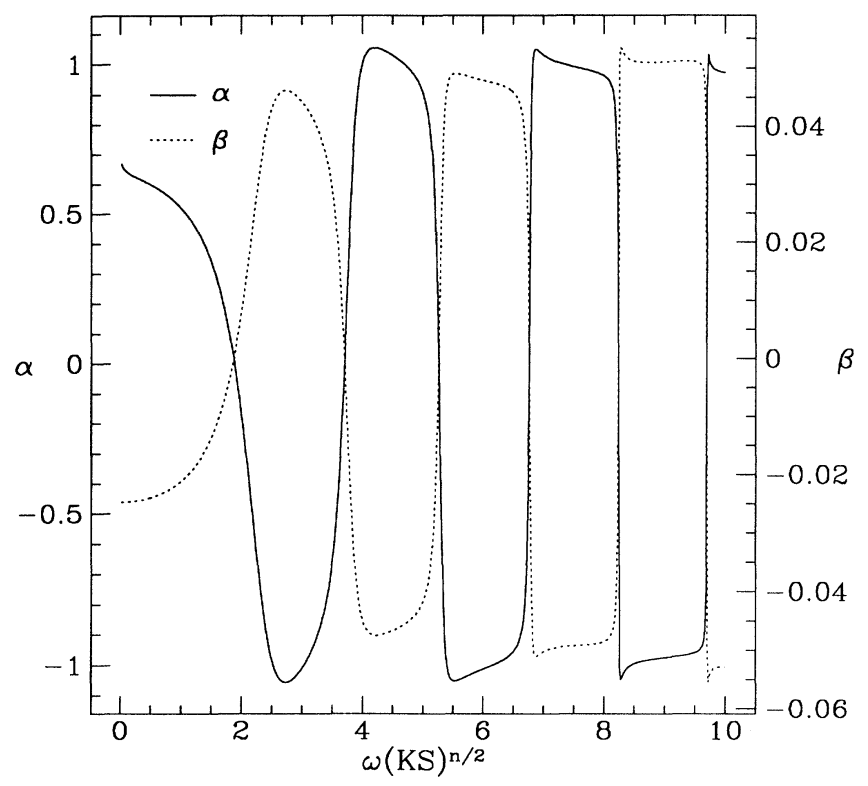

FIG. 8. Coefficients $\alpha$ and $\beta$ for a $\Gamma=1.5, \widetilde{q}_{c}=10^{-2}$ polytrope. Notice that the derivatives of these functions can be quite difficult to evaluate numerically, especially for large $\omega$.

the function $\left(\alpha^{2}+\beta^{2}\right)^{-1 / 2}$ and correspond to the real parts of the eigenfrequencies $\omega_{n}$.

Figure 9 shows a resonance spectrum obtained by plotting the quantity $\left(\alpha^{2}+\beta^{2}\right)^{-1 / 2}$ vs $\omega$. The position of each peak in the spectrum corresponds to the real part of one of the eigenfrequencies $\omega_{n}$, and the width of that peak corresponds to the imaginary part of that eigenfrequency.

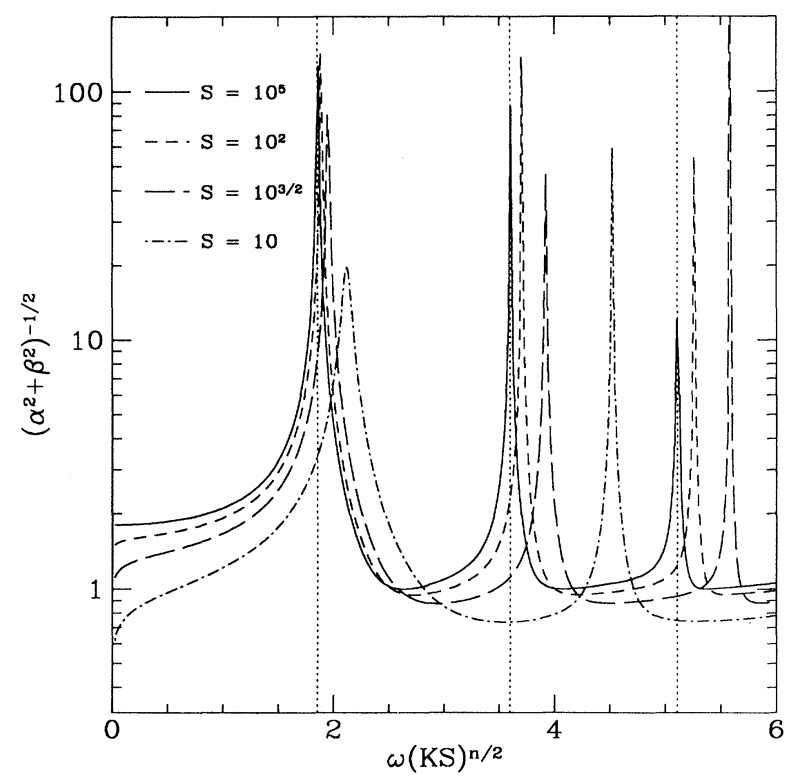

FIG. 9. Frequency spectrum of a $\Gamma=1.5(n=2)$ cylindrical polytrope shown for several values of $S=\widetilde{q}_{c}^{-1}$. The eigenfrequencies $\omega_{n}$ in the Newtonian limit are proportional to $(K S)^{-n / 2}$, and are shown on this plot as vertical dotted lines.
Notice that as $\rho_{c}$ approaches zero, the real parts of $\omega_{n}$ approach their Newtonian values: For sufficiently small $\rho_{c}$, the real parts of $\omega_{n}$ agree with the Newtonian limit to at least one part in $10^{5}$.

The imaginary parts of $\omega_{n}$, as determined from Eq. (30), are all positive, and in addition, they tend towards zero in the nonrelativistic limit, as one can see by examining the widths of the peaks in Fig. 9. The fact that the eigenfrequencies $\omega_{n}$ become purely real in the nonrelativistic limit simply indicates that gravitational radiation is very weak, and hence there is very little damping of the perturbations. Note that it becomes increasingly difficult to determine $\omega_{n}$ as $\rho_{c}$ becomes small and/or $\widetilde{\omega}$ grows the calculation of $\alpha^{\prime}$ and $\beta^{\prime}$ for use in Eq. (30) becomes dominated by roundoff error because one is forced to evaluate $\alpha$ and $\beta$ for values of $\omega$ that differ by an extremely small amount (see Fig. 8).

Once the eigenvalues for the single value of $\rho_{c}$ and $\Gamma$ have been located by the resonance method, it is possible to trace the behavior of a single eigenvalue as a function of $\rho_{c}$ or $\Gamma$ using the complex search method. The idea is simple: change $\rho_{c}$ or $\Gamma$ by a small amount, and use the previous value of $\omega_{n}$ as an initial guess for the next value. To take larger steps in $\rho_{c}$ or $\Gamma$, use the two or three previous values of $\omega_{n}$ to extrapolate to an initial guess for the next value of $\omega_{n}$. Figure 10 shows the fundamental eigenvalue $\bar{\omega}_{0} \equiv \bar{\omega}_{r}+i \bar{\omega}_{i}$ vs $\rho_{c}$ for two different values of $\Gamma$, as obtained by this method. Since the imaginary part $\bar{\omega}_{i}$ is positive, the fundamental quasinormal mode is exponentially damped in time. Furthermore, in the ultrarelativistic limit $\bar{\omega}_{0}$ approaches a constant value, in agreement with the scaling law (49). This asymptotic behavior indicates that $\bar{\omega}_{i}$ remains positive for arbitrarily large $\rho_{c}$.

For all values of $\Gamma$ and $\rho_{c}$ considered, the imaginary

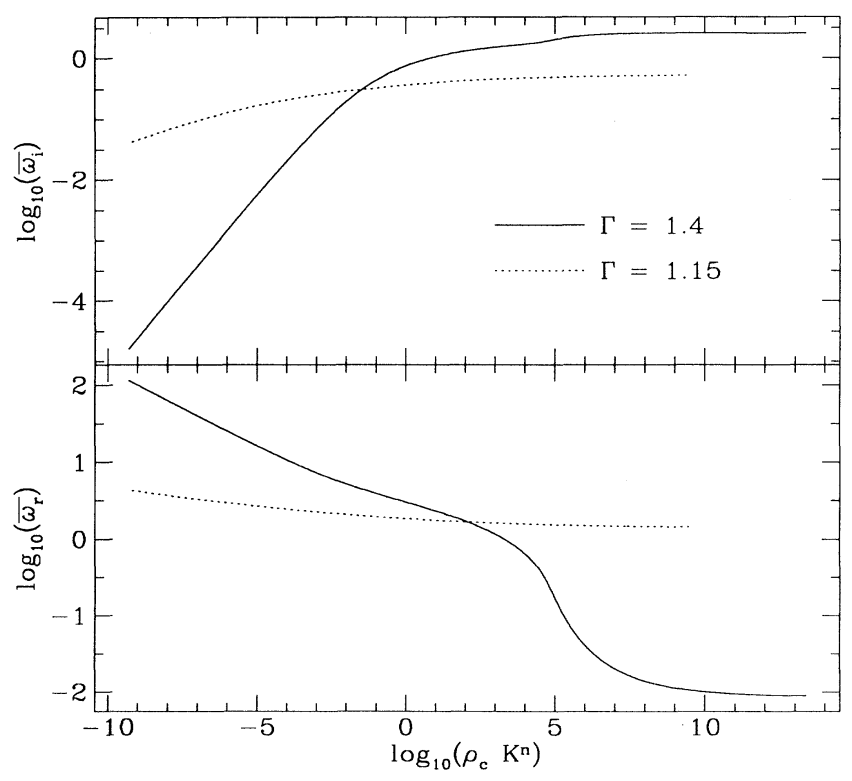

FIG. 10. Fundamental eigenvalue $\bar{\omega}_{0} \equiv \bar{\omega}_{r}+i \bar{\omega}_{i}$ as a function of $\rho_{c}$ for two values of $\Gamma$. 
part of each eigenvalue computed turned out to be positive. Of course, there is no way to check every eigenvalue of every configuration, but the fact that no negative imaginary parts were found suggests that equilibrium cylindrical polytropes are stable with respect to radial pulsations, in accordance with the result obtained from the $C$-energy method.

\section{CONCLUSION}

There are at least four ways in which the behavior of infinite cylinders differs from that of finite systems: First of all, collapse of an infinite cylinder never produces a black hole. Either a naked singularity appears, or else the cylinder bounces. Second, an infinitesimal amount of rotation always halts the collapse of an infinite cylinder to a singularity [6], but it does not halt the collapse of a finite object to a Kerr black hole. Third, pressure always causes a collapsing cylinder to bounce [7], whereas pressure in a finite spherical system can actually accelerate the collapse to a black hole and central singularity. Finally, as shown in this paper, all polytropic infinite cylinders are stable against radial perturbations, whereas for spherical polytropes pressure regeneration causes a configuration of any index $\Gamma$ to collapse if it is sufficiently relativistic.

These differences suggest that one cannot always use an infinite cylinder to gain physical insight into the collapse of a nonspherical asymptotically flat system. Any such attempt, even if it incorporates realistic physics such as pressure or rotation, contains the unrealistic assumptions of an infinite configuration and a metric that diverges at infinity. These unrealistic assumptions seem to qualitatively change the nature of catastrophic gravitational collapse, and may invalidate the application to asymptotically flat spacetimes.

\section{ACKNOWLEDGMENTS}

This research was supported in part by NSF Grant Nos. AST 91-19475 and PHY 90-07834 and NASA Grant No. NAGW-2364 at Cornell University.

\section{APPENDIX A: DERIVATION [12] OF EQUATION (30)}

For any complex value of $\omega$, there exists a solution to Eqs. (20) and (21) that is unique up to an arbitrary multiplicative complex constant. Such a solution will have the form (27) outside of the star, and in general both $C_{\text {in }}$ and $C_{\text {out }}$ will be nonzero. If one chooses $\omega$ to be real, then the arbitrary constant can be chosen so that $\delta \psi$ and $\xi$ are real everywhere. Call the perturbation functions for this solution $\delta \psi_{0}$ and $\xi_{0}$. In this case, Eq. (27) for the field perturbation in the vacuum can be written in the form

$$
\delta \psi_{0}=\alpha(\omega) J_{0}\left(r^{*}\right)-\beta(\omega) N_{0}\left(r^{*}\right)
$$

where $J_{0}$ and $N_{0}$ are Bessel functions, and $r^{*} \equiv \omega\left|B_{s}\right| r$.

If $\omega$ is complex, then Eqs. (20) and (21) can be written as four equations for the real and imaginary parts of $\delta \psi$ and $\xi$ :

$$
\begin{aligned}
\xi_{r}^{\prime \prime} \Gamma p= & \xi_{r}\left[X_{1}-B^{2}\left(\omega_{r}^{2}-\omega_{i}^{2}\right)(p+\rho)\right]+\xi_{r}^{\prime} X_{2}+\delta \psi_{r} X_{3} \\
& +\delta \psi_{r}^{\prime} X_{4}-2 B^{2} \xi_{i} \omega_{i} \omega_{r} \\
-\delta \psi_{r}^{\prime \prime}= & \xi_{r} Y_{1}+\xi_{r}^{\prime} Y_{2}+\delta \psi_{r}\left[B^{2}\left(\omega_{r}^{2}-\omega_{i}^{2}\right)+Y_{3}\right]+\delta \psi_{r}^{\prime} Y_{4} \\
& -2 B^{2} \delta \psi_{i} \omega_{r} \omega_{i} \\
\xi_{i}^{\prime \prime} \Gamma p= & \xi_{i}\left[X_{1}-B^{2}\left(\omega_{r}^{2}-\omega_{i}^{2}\right)(p+\rho)\right]+\xi_{i}^{\prime} X_{2}+\delta \psi_{i} X_{3} \\
& +\delta \psi_{i}^{\prime} X_{4}+2 B^{2} \xi_{r} \omega_{i} \omega_{r} \\
-\delta \psi_{i}^{\prime \prime}= & \xi_{i} Y_{1}+\xi_{i}^{\prime} Y_{2}+\delta \psi_{i}\left[B^{2}\left(\omega_{r}^{2}-\omega_{i}^{2}\right)+Y_{3}\right]+\delta \psi_{i}^{\prime} Y_{4} \\
& +2 B^{2} \delta \psi_{r} \omega_{r} \omega_{i}
\end{aligned}
$$

where

$$
\begin{aligned}
& \delta \psi \equiv \delta \psi_{r}+i \delta \psi_{i} \\
& \xi \equiv \xi_{r}+i \xi_{i} \\
& \omega \equiv \omega_{r}+i \omega_{i} .
\end{aligned}
$$

If $\omega_{i}<<\omega_{r}$, the arbitrary constant can be chosen so that $\xi_{i}$ and $\delta \psi_{i}$ are of order $\omega_{i}$ or smaller. (This follows from the fact that for $\omega_{i}=0$, one can choose $\xi$ and $\delta \psi$ to be real.) One can therefore define $\Xi \equiv \xi_{i} / \omega_{i}$ and $\Psi_{i} \equiv \delta \psi_{i} / \omega_{i}$. If one neglects all terms of order $\omega_{i}^{2}$ in Eqs. (A2)-(A5), the result is

$$
\begin{aligned}
\xi_{r}^{\prime \prime} \Gamma p= & \xi_{r}\left[X_{1}-B^{2} \omega_{r}^{2}(p+\rho)\right]+\xi_{r}^{\prime} X_{2}+\delta \psi_{r} X_{3}+\delta \psi_{r}^{\prime} X_{4}, \\
-\delta \psi_{r}^{\prime \prime}= & \xi_{r} Y_{1}+\xi_{r}^{\prime} Y_{2}+\delta \psi_{r}\left(B^{2} \omega_{r}^{2}+Y_{3}\right)+\delta \psi_{r}^{\prime} Y_{4}, \\
\Xi^{\prime \prime} \Gamma p= & \Xi\left[X_{1}-B^{2} \omega_{r}^{2}(p+\rho)\right]+\Xi^{\prime} X_{2}+\Psi X_{3}+\Psi^{\prime} X_{4} \\
& +2 B^{2} \xi_{r} \omega_{r}, \\
-\Psi^{\prime \prime}= & \Xi Y_{1}+\Xi^{\prime} Y_{2}+\Psi\left(B^{2} \omega_{r}^{2}+Y_{3}\right)+\Psi^{\prime} Y_{4}+2 B^{2} \Psi \omega_{r} .
\end{aligned}
$$

Since Eqs. (A7) and (A8) are identical to Eqs. (20) and (21) for real $\omega$, and they satisfy the same boundary conditions in the limit $\omega_{i}<<\omega_{r}$, one may choose as the solutions of Eqs. (A7) and (A8) the functions $\xi_{0}$ and $\delta \psi_{0}$. Therefore, one can write, for the real part of the field perturbation outside the star,

$$
\delta \psi_{r}=\alpha\left(\omega_{r}\right) J_{0}\left(r^{*}\right)-\beta\left(\omega_{r}\right) N_{0}\left(r^{*}\right),
$$

where $r^{*} \equiv \omega_{r}\left|B_{s}\right| r$. If one writes $\delta \psi_{r}$ and $\xi_{r}$ as $\delta \psi_{r}\left(r, \omega_{r}\right)$ and $\xi_{r}\left(r, \omega_{r}\right)$ to emphasize the dependence of these quantities on $\omega_{r}$, then one can see that

$$
\begin{aligned}
& \Xi(r)=\frac{\partial \xi_{r}\left(r, \omega_{r}\right)}{\partial \omega_{r}}, \\
& \Psi(r)=\frac{\partial \delta \psi_{r}\left(r, \omega_{r}\right)}{\partial \omega_{r}}
\end{aligned}
$$

are solutions to Eqs. (A4) and (A5) and that these solutions satisfy the same boundary conditions as $\xi_{r}$ and $\delta \psi_{r}$.

Therefore, one can write, for the field perturbation outside the star, 


$$
\begin{aligned}
\delta \psi= & \delta \psi_{r}+i \omega_{i} \Psi \\
= & \left(\alpha+i \omega_{i} \alpha^{\prime}\right) J_{0}\left(r^{*}\right)-\left(\beta+i \omega_{i} \beta^{\prime}\right) N_{0}\left(r^{*}\right) \\
& +i \omega_{i} r\left|B_{s}\right|\left[-\alpha J_{1}\left(r^{*}\right)+\beta N_{1}\left(r^{*}\right)\right],
\end{aligned}
$$

where primes denote differentiation with respect to $\omega_{r}$. For $\omega_{i} \ll \omega_{r}$, one can neglect $\left|B_{s}\right| r J_{1} \alpha$ and $\left|B_{s}\right| r N_{1} \beta$ compared to $J_{0} \alpha^{\prime}$ and $N_{0} \beta^{\prime}$, since $\alpha^{\prime}$ and $\beta^{\prime}$ are much larger than $\alpha$ and $\beta$ near an eigenfrequency, as shown in Fig. 8. In this approximation, Eq. (A14) can be rewritten in the form

$$
\begin{aligned}
& \delta \psi=\frac{1}{2}\{ {\left[\left(\alpha-\omega_{i} \beta^{\prime}\right)+i\left(\beta+\omega_{i} \alpha^{\prime}\right)\right] H_{0}^{(1)}\left(r^{*}\right) } \\
&\left.+\left[\left(\alpha+\omega_{i} \beta^{\prime}\right)+i\left(\omega_{i} \alpha^{\prime}-\beta\right)\right] H_{0}^{(2)}\left(r^{*}\right)\right\} .
\end{aligned}
$$

The $H_{0}^{(1)}$ term is the one that describes incoming waves; this term must vanish if one requires that only outgoing waves are present. Hence, one must have

$$
\omega_{i}=\alpha / \beta^{\prime}=-\beta / \alpha^{\prime}
$$

if there are no incoming gravitational waves. Therefore, by examining the behavior of the real solutions of Eqs. (20) and (21) for real $\omega$ and imposing no particular condition on the gravitational waves outside the cylinder, one can determine the discrete complex eigenvalues $\omega_{n}$ that satisfy these same equations subject to the condition that there exist only outgoing gravitational waves at infinity.

\section{APPENDIX B: DERIVATION OF FIELD EQUATIONS}

Adopt a line element of the form

$d s^{2}=-e^{2 v} d t^{2}+e^{2(\gamma-\psi)} d r^{2}+e^{2 \psi} d z^{2}+r^{2} e^{-2 \psi} d \phi^{2}$,

where $v, \gamma$, and $\psi$ are functions of $r$ and $t$. For algebraic convenience, it is useful to define the function

$$
\alpha \equiv \gamma-\psi
$$

The nonvanishing components of the Einstein tensor are

$$
\begin{aligned}
& G_{\hat{t}}^{\hat{t}}=e^{-2 \alpha}\left[\psi^{\prime 2}-\frac{\alpha^{\prime}+\psi^{\prime}}{r}\right]+\dot{\psi}^{2} e^{-2 v}, \\
& G_{p}^{\hat{t}}=e^{-(\alpha+v)}\left[2 \psi^{\prime} \dot{\psi}-\frac{\dot{\alpha}+\dot{\psi}}{r}\right], \\
& G_{\widehat{r}}^{\hat{P}}=e^{-2 \alpha}\left(\frac{v^{\prime}+\psi^{\prime}}{r}-\psi^{\prime 2}\right)-\dot{\psi}^{2} e^{-2 v}, \\
& \begin{aligned}
G_{\hat{z}}^{\hat{z}}=e^{-2 \alpha} & {\left[v^{\prime \prime}-\psi^{\prime \prime}+v^{\prime 2}+\frac{v^{\prime}-2 \psi^{\prime}-\alpha^{\prime}}{r}\right.} \\
& \left.+\left(\alpha^{\prime}+\psi^{\prime}\right)\left(\psi^{\prime}-v^{\prime}\right)\right]^{r}
\end{aligned} \\
& +e^{-2 v}\left[\ddot{\psi}-\dot{\psi}(\dot{\psi}+\dot{v}-\dot{\alpha})-\ddot{\alpha}-\dot{\alpha}^{2}+\dot{\alpha} \dot{v}\right], \\
& G_{\hat{\phi}}^{\hat{\phi}}=e^{-2 \alpha}\left[v^{\prime \prime}+\psi^{\prime \prime}+v^{\prime 2}+v^{\prime} \psi^{\prime}+\psi^{\prime 2}-\alpha^{\prime}\left(\psi^{\prime}+v^{\prime}\right)\right] \\
& +e^{-2 v}\left[-\ddot{\psi}-\dot{\psi}(\dot{\psi}-\dot{v}+\dot{\alpha})-\ddot{\alpha}-\dot{\alpha}^{2}+\dot{\alpha} \dot{v}\right],
\end{aligned}
$$

where

$$
\begin{aligned}
& A \equiv 8 \pi r e^{2 \alpha}, \\
& y \equiv B+1 \equiv 1-e^{\alpha-v} .
\end{aligned}
$$

Subtracting Eqs. (B8) and (B9), and using the equilibrium equations, one obtains

$$
\frac{\left(r \delta \psi^{\prime}\right)^{\prime}}{r}+\frac{B}{2 r}\left(\delta v^{\prime}-\delta \alpha^{\prime}\right)+\delta \psi^{\prime}(p-\rho) A+\omega^{2} B^{2} \delta \psi=0 .
$$


Other useful relations can be found directly from energy-momentum conservation: From the equation $u_{\alpha} T_{; \beta}^{\alpha \beta}=0$ one obtains

$$
\delta \rho=-\xi \rho^{\prime}-(p+\rho)\left[\delta \alpha+\frac{e^{-\alpha}}{r}\left(r \xi e^{\alpha}\right)^{\prime}\right] .
$$

If we have an adiabatic equation of state $P=K \rho_{0}^{\Gamma}$, then adiabatic perturbations must satisfy

$$
\frac{\delta p+\xi p^{\prime}}{p}=\Gamma \frac{\delta \rho+\xi \rho^{\prime}}{p+\rho}
$$

which implies

$$
\delta p=-\xi p^{\prime}-\Gamma p\left[\delta \alpha+\frac{e^{-\alpha}}{r}\left(r \xi e^{\alpha}\right)^{\prime}\right] .
$$

In addition, the Euler equation (B5) gives to first order

$$
(p+\rho)\left(-\omega^{2} B^{2} \xi+\delta v^{\prime}\right)+(\delta p+\delta \rho) v^{\prime}+\delta p^{\prime}=0 .
$$

Finally, by suitably combining Eqs. (B16), (B13), (B15), (B10), (B6), and (B7), one obtains the perturbation equation (20), and by suitably combining Eqs. (B12), (B10), (B13), (B15), (B6), and (B7), one obtains the perturbation equation (21).
[1] S. L. Shapiro and S. A. Teukolsky, Phys. Rev. Lett. 66, 994 (1991); Am. Sci. 79, 330 (1991); Phys. Rev. D 45, 2206 (1992).

[2] K. S. Thorne, in Magic Without Magic: John Archibald Wheeler, edited by J. Klauder (Freeman, San Francisco, 1972), p. 231.

[3] C. W. Misner, K. S. Thorne, and J. A. Wheeler, Gravitation (Freeman, San Francisco, 1973).

[4] R. Penrose, Nuovo Cimento 1, 252 (1969).

[5] F. Echeverria, Phys. Rev. D 47, 2271 (1993).

[6] T. A. Apostolatos and K. S. Thorne, Phys. Rev. D 46 , 2435 (1992).

[7] T. Piran, Phys. Rev. Lett. 41, 1085 (1978).
[8] See, e.g., S. L. Shapiro and S. A. Teukolsky, Black Holes, White Dwarfs, and Neutron Stars (Wiley, New York, 1983).

[9] K. S. Thorne, Ph.D. thesis, Princeton University, 1965; available from University Microfilms Inc., Ann Arbor, Michigan.

[10] T. Levi-Civita, Rend. Acc. Lincei 28, 101 (1919).

[11] S. Chandrasekhar, Phys. Rev. Lett. 12, 114 (1964); 12, 437 (1964).

[12] This derivation closely follows that of S. Chandrasekhar, V. Ferrari, and R. Winston, Proc. R. Soc. London A434, 635 (1991). 